\title{
Pairing JAK with MEK for improved therapeutic efficiency in myeloproliferative disorders
}

\author{
David A. Williams
}

Harvard Medical School, Dana-Farber/Boston Children's Cancer and Blood Disorders Center, Boston, Massachusetts, USA.

\begin{abstract}
The identification of JAK2 mutations as disease-initiating in myeloproliferative neoplasms (MPNs) has led to new and effective therapies for these diseases. In a study published in this issue of the JCI, Stivala et al. explored the key observation that JAK inhibition successfully suppresses MAPK activation in MPN cell lines and primary MPN cells in vitro, and the finding that it failed to completely and effectively suppress MAPK activation in vivo in two mouse models. The authors went on to show that dual inhibition of JAK and the MAP kinase pathway provided enhanced therapeutic efficacy in the in vivo models of MPN.
\end{abstract}

Inhibitors of JAK in myeloproliferative neoplasms Myeloproliferative neoplasms (MPNs) are disorders of hematopoietic stem cells associated with activated JAK/STAT signaling and present with chronic excessive production of myeloid cells. These include excessive red cell production as seen in polycythemia vera (PV), excessive platelet production as in essential thrombocythemia (ET), and excessive megakaryocytes and bone marrow fibrosis as in myelofibrosis (MF) (1). These chronic conditions have a risk of further transformation to acute leukemias that are typically highly refractory to chemotherapy. Currently, the only therapy with curative potential for MPN is hematopoietic stem cell transplantation (HSCT); however, this approach is limited by regimen-related toxicities that are particularly prevalent in the older population, the group most affected by MPNs. Identification of JAK2 mutations (2-5) as disease-initiating in MPN has led to new and effective therapies for these diseases. For instance, ruxolitinib, a molecularly targeted inhibitor of JAK1/JAK2, is now FDA approved for the treatment of intermediate- or high-risk myelofibrosis and PV in patients who are resistant to or intolerant of hydroxyurea, one of the most commonly used agents in MPN patients.

JAK2 is a non-receptor tyrosine kinase that is required for normal blood cell development. The efficacy of current JAK2 inhibitors is limited. Current JAK2 inhibitors are not strongly selective for the JAK2-mutant cells, and, as a result, molecular remissions are not common (6). Furthermore, a substantial portion of patients with myelofibrosis come off treatment as a result of disease progression and/or toxicity. Earlier work has suggested that a mechanism of resistance to JAK2 inhibitors is a result of continued dysregulation in JAK2 activation and downstream signaling activities (7). In addition to constitutive activating JAK2 mutations, mutations in the thrombopoietin receptor $M P L$ and in the ER chaperone protein calreticulin $(C A L R)$ are diseaseinitiating in MPNs, and each of these so-called MPN phenotypic driver mutations leads to activation of MPL/JAK2 cytokine signaling (8), resulting in the activation of downstream targets including STATs, AKT/PI3K, and the MAPK pathway (Figure 1). The MAPK pathway is complex and, importantly, downstream of RAS, a GTPase

\section{Related Article: p. 1596}

Conflict of interest: The author has declared that no conflict of interest exists.

implicated in a large fraction of cancers and some leukemias (9). The MAPK pathway includes the kinases RAF, ERK1/2, and MEK1/2, with activated ERK leading to specific activation of downstream MEK as a target. Inhibitors of this pathway are in clinical development for other cancers, including $R A S$-mutated leukemias.

\section{Boosting JAK inhibition for effective treatment of myeloproliferative neoplasms} In a study published in this issue of the JCI, Stivala et al. (10) explore a key observation that ruxolitinib treatment inhibited JAK/ STAT and ERK activation in MPN cell lines and even primary MPN cells in vitro but failed to completely inhibit ERK activation effectively in vivo in two mouse models. They noted in particular that JAK2 inhibition in vivo failed to effectively inhibit the activation of the MAPK pathway, and surmised that this difference could represent a non-cell-autonomous effect of the in vivo tumor microenvironment and, importantly, offer an additional therapeutic target. The authors went on to show that dual inhibition of JAK and the MAPK pathway prevented the persistent activation of MEK in the presence of JAK2 inhibition in vivo and provided enhanced therapeutic efficacy in the in vivo models of MPN. This was particularly noteworthy for the myelofibrosis associated with MPN.

Mechanistically, using orthogonal approaches of mRNA and protein analysis, the authors demonstrated that PDGFR $\alpha$ is activated in these in vivo MPN models and that activated PDGFR $\alpha$ is responsible for persistent MEK activation in the presence of effective JAK inhibition. Interestingly, PDGF, which signals through PDGFR $\alpha$, is present in the hematopoietic microenvironment of the bone marrow and has been previously implicated in the development of myelofibrosis (11), providing a satisfying potential mechanistic linkage to the efficacy seen with inhibition of the MAPK pathway and the myelofibrosis 
A

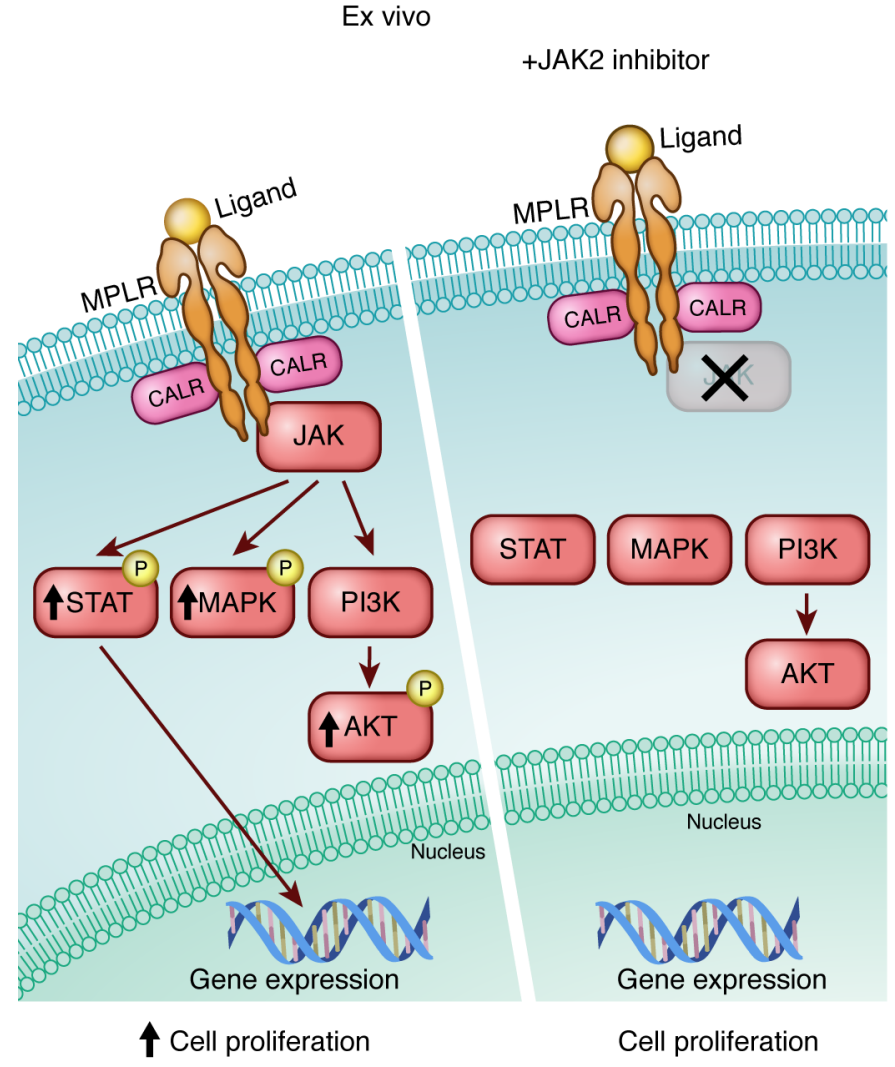

C

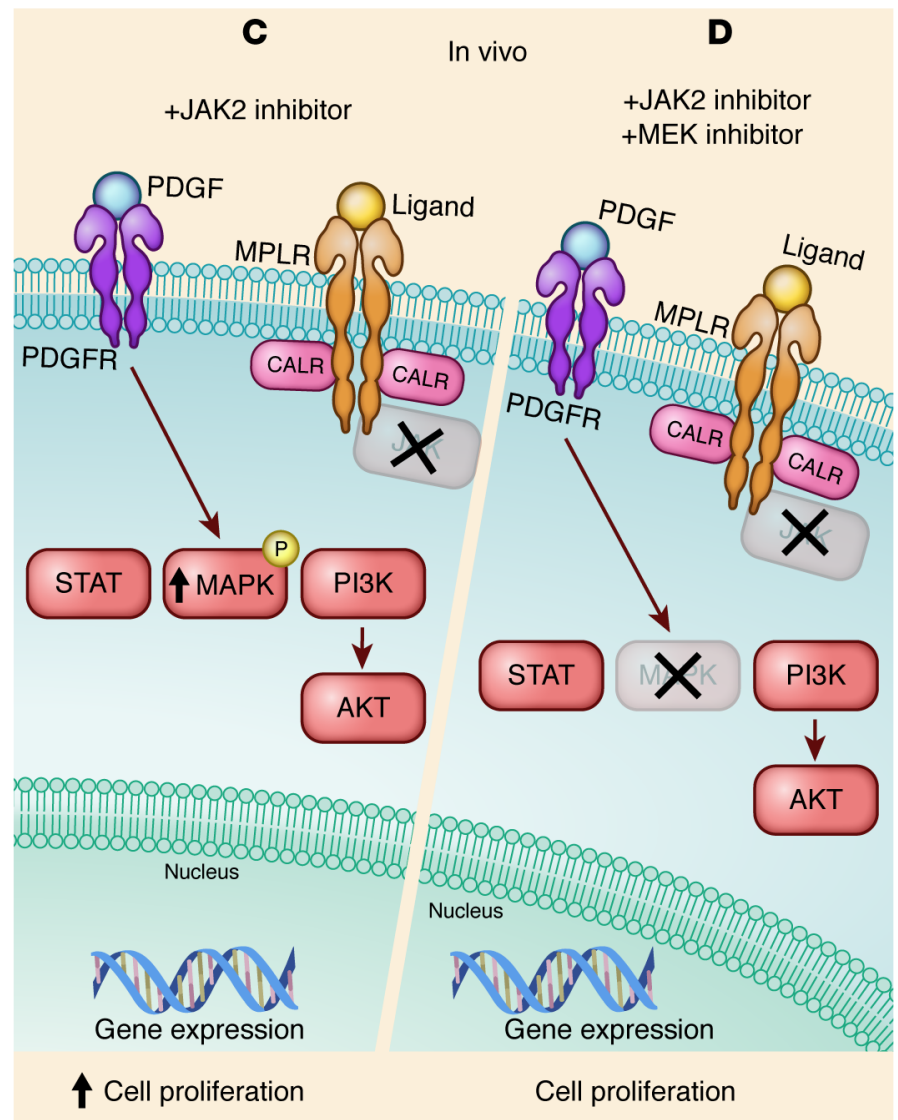

Figure 1. Inhibiting the JAK pathway in MPN. (A and B) Ex vivo inhibition strategy. Activation of the JAK pathway leads to enhanced cell proliferation, a hallmark of MPN. JAK2 inhibition ex vivo inhibits MEK/ERK signaling, ultimately leading to a reduction in cell proliferation. (C and D) In vivo inhibition strategy. JAK2 inhibitors are not able to induce molecular remissions in vivo. JAK2-independent compensatory activation of the MEK/ERK signaling pathway is attributed to activated PDGFR. Combined targeting of JAK2 and MEK inhibits ERK activation and reduces cell proliferation in vivo. MPLR, thrombopoietin receptor. Figure illustrated by Rachel Davidowitz.

phenotype of MPN. The authors demonstrated persistent levels of PDGF even with effective JAK2 inhibition in vivo, supporting its potential role in the activation of the MAPK pathway in MPN and the lack of efficacy of monotherapy with JAK2 inhibition.

\section{JAK/ERK inhibition: a roadmap for a wider cancer treatment regimen}

Overall, these studies suggest that compensatory ERK activation limits the efficacy of JAK2 inhibition in MPN models in vivo and that dual JAK/MEK inhibition provides an opportunity for improved therapeutic efficacy. These studies imply that additional knowledge of targeted therapies can be ascertained from in vivo models and these may be critical to understanding resistant disease or relapse in the setting of these therapies. Several unanswered questions remain to be explored. A key one is whether combination therapy, when applied to humans with MPNs, is associated with increased efficacy and/or is limited by excessive side effects. In addition, the cell-autonomous versus tumor microenvironment role of PDGF, which is expressed in both hematopoietic cells and bone marrow stromal cells, is not yet completely clear. Finally, the potential role of compensatory ERK/MEK signaling as a more general mechanism of tumor resistance to targeted therapies in other cancers needs to be studied to determine the relevance of these findings in other clinical situations with unmet medical need.

\section{Acknowledgments}

I thank Ann Mullally and Scott Armstrong for helpful discussions.

Address correspondence to: David A. Williams, Leland Fikes Chair of Pediatrics, Harvard Medical School, Dana-Farber/Boston Children's Cancer and Blood Disorders Center, 300 Longwood Ave. Karp 08125.3,
Boston, Massachusetts 02115, USA. Phone: 617.919.2697; Email: DAWilliams @childrens.harvard.edu.

1. Spivak JL. Myeloproliferative neoplasms. $N$ Engl JMed. 2017;377(9):895-896.

2. Kralovics R, et al. A gain-of-function mutation of JAK2 in myeloproliferative disorders. $N$ Engl J Med. 2005;352(17):1779-1790.

3. Levine RL, et al. Activating mutation in the tyrosine kinase JAK2 in polycythemia vera, essential thrombocythemia, and myeloid metaplasia with myelofibrosis. Cancer Cell. 2005;7(4):387-397.

4. Baxter EJ, et al. Acquired mutation of the tyrosine kinase JAK2 in human myeloproliferative disorders. Lancet. 2005;365(9464):1054-1061.

5. James C, et al. A unique clonal JAK2 mutation leading to constitutive signalling causes polycythaemia vera. Nature. 2005;434(7037):1144-1148.

6. Mead AJ, Mullally A. Myeloproliferative neoplasm stem cells. Blood. 2017;129(12):1607-1616.

7. Koppikar P, et al. Heterodimeric JAKSTAT activation as a mechanism of persistence to JAK2 inhibitor therapy. Nature. 2012;489(7414):155-159.

8. Rampal R, et al. Integrated genomic analysis 
illustrates the central role of JAK-STAT pathway activation in myeloproliferative neoplasm pathogenesis. Blood. 2014;123(22):e123-e133.

9. Burotto M, Chiou VL, Lee JM, Kohn EC. The MAPK pathway across different malignancies: a new perspective. Cancer. 2014;120(22):3446-3456.

10. Stivala S, et al. Targeting compensatory MEK/ ERK activation increases JAK inhibitor efficacy in myeloproliferative neoplasms. JClin Invest.
2019;129(4):1596-1611.

11. Demoulin JB, Montano-Almendras CP. Plateletderived growth factors and their receptors in normal and malignant hematopoiesis. Am J Blood Res. 2012;2(1):44-56. 